Díaz-Serrano, J., Alfageme-Gónzalez, M.B. \& Cutanda-López, M.T. (2022). Interacción del rendimiento académico con los estilos de aprendizaje y de enseñanza. Revista Electrónica Interuniversitaria de Formación del Profesorado, 25(1), 145-160.

DOI: https://doi.org/10.6018/reifop.486081

\title{
Interacción del rendimiento académico con los estilos de aprendizaje y de enseñanza
}

José Díaz Serrano, María Begoña Alfageme González, María Trinidad Cutanda López

Universidad de Murcia

\section{Resumen}

Se plantea evaluar la incidencia de los estilos de aprendizaje sobre el rendimiento de los estudiantes en contenidos geográficos. Muestra de diez centros educativos: 21 docentes de Geografía e Historia y 483 estudiantes de secundaria, con un perfil de aprendizaje en el que predominan los estilos Reflexivo y Teórico. Obtienen mejores calificaciones frente a contenidos geográficos cuando su perfil de estilos de aprendizaje presenta puntuaciones más altas en Reflexivo y Teórico y más bajas en Activo. Las calificaciones son mejores cuando estudiante y docente coinciden en poseer una preferencia alta por los estilos Reflexivo y Formal, respectivamente.

\section{Palabras clave}

Ciencias Sociales; Educación Secundaria; Estilos de Aprendizaje; Estilos de Enseñanza.

\section{Interaction of academic performance with learning and teaching styles}

\begin{abstract}
We propose to evaluate the incidence learning styles on student performance in geographic content. The sample of ten educational centers: 21 Geography and History teachers and 483 high school students, with a learning profile in which the Reflective and Theoretical styles predominate. Students obtain better scores against geographic contents when their learning styles profile shows higher scores in Reflective and Theoretical and lower scores in Active.
\end{abstract}


The grades are better when the student and the teacher coincide in having a high preference for Reflective and Formal styles, respectively.

\section{Key words}

Social Science; Secondary Education; Styles of Learning; Styles of Teaching.

\section{Introducción}

Las teorías de estilos de aprendizaje y de enseñanza y su diagnóstico para orientar las prácticas de aula, han tenido un amplio desarrollo desde finales del siglo XX en la comunidad educativa (Stobart, 2010). A la luz de las mismas, se considera que la enseñanza hoy no pasa únicamente por el dominio del contenido por parte del profesorado, la organización del discurso conceptual y la propuesta de tareas. También, requiere conocer cómo aprende el alumnado y cómo podemos facilitar su aprendizaje. En consonancia, el docente debe, entre otros: ser flexible adecuando su planificación conforme a la dinámica del grupo-clase, atender a la diversidad de ritmos de aprendizaje, intereses y experiencias, y a las distintas formas de procesar la información que identifican un determinado estilo de aprendizaje (Laudadío y Mazzitelli, 2019; Pérez, Leyva, Ocampo y De Luna, 2018). O, en otros términos, cada estudiante tiene preferencia por una determinada estrategia de aprendizaje que cognitivamente se manifiesta en un estilo de aprendizaje. Si lo que se pretende es ofrecer una enseñanza más individualiza, el docente habrá de poner en práctica sus estrategias de enseñanza atendiendo a las características del grupo de estudiantes y la relación particular de cada uno de ellos con su aprendizaje. No en vano, la forma en la que cada docente interactúa con el alumnado y organiza su enseñanza, genera un estilo propio y único (Martínez Geijo, 2009; Renés y Martínez, 2015; Isaza y Henao, 2012), que ofrece al alumnado una experiencia educativa diferente (Lambert y Balderstone, 2010).

De acuerdo con Alonso, Gallego y Honey (1999), se alude a estilos cognitivos y no a estilos de aprendizaje omitiendo con ello el debate existente en torno a éstos últimos, que ha venido obstaculizando el progreso de aplicación teórica en contextos educativos. En tal sentido, retoman el carácter puente de la dimensión cognitiva del aprendizaje incluyendo los estudios de Psicología cognitiva que tratan sobre la diferencia en los sujetos respecto a las formas de conocer. Junto con Camana y Torres (2018) y Coto (2020), asumen la definición de Keefe (en Camana y Torres, 2018, p- 3) que define los estilos de aprendizaje como los "rasgos cognitivos, afectivos y fisiológicos que operan como posibles indicadores de cómo un individuo promedio percibe, apropia e interactúa o responde a una determinada información".

Para Renés y Martínez (2015), el modelo de estilos de aprendizaje de Alonso y sus colaboradores, parten de las teorías del aprendizaje de tipo cognitivo que, desde principios de siglo XX, entienden el comportamiento como el producto de la interacción entre la persona y su ambiente psicológico cercano e inmediato. Consideran así el aprendizaje como proceso mediante el cual, el conocimiento se crea a través de la transformación de la experiencia (Kolb, 1984). Y, aceptan la división del proceso de aprendizaje en etapas cíclicas que van a estar influidas por experiencias vividas, circunstancias medio-ambientales y la herencia (Ruiz, Trillos y Morales, 2006). A partir de tales experiencias, se configuran preferencias cognitivas a la hora de percibir y procesar la información, identificadas como estilos de aprendizaje. En suma, lo ideal sería que todo el mundo fuera capaz de experimentar, reflexionar, elaborar hipótesis y aplicar a partes iguales. Sin embargo, los individuos son más capaces de una cosa que de otra (Alonso et al., 1999) 
El trabajo que aquí se presenta, tienen en consideración la diversidad de enfoques sobre estilos de aprendizaje (Nivela-Cornejo, Echeverría-Desiderio y Morillo, 2019), partiendo del diagnóstico del estilo de aprendizaje en aquellos procesos educativos que tienen lugar en las aulas de la Educación Secundaria, en particular, en el área de Geografía. Entre ellos, por su especial interés para el estudio, se consideran aquellos modelos de mayor continuidad y aplicación de la realidad escolar en nuestro contexto (Delgago, Martínes y Ponce, 2016): los de Kolb (1984) y Honey y Mumford (1988) que, a raíz del trabajo de Alonso et al. (1999), han sido la base de la fundamentación y operativización del cuestionario CHAEA (Cuestionario Honey-Alonso de Estilos de Aprendizaje). Un instrumento de diagnóstico (Figura 1), que mantiene similar estructura y aplicación al de Honey y Mumford (1988), y su taxonomía de estilos de aprendizaje en el ciclo experiencial.

Figura 1.

Estilos de aprendizaje y descriptores del aprendizaje experiencial

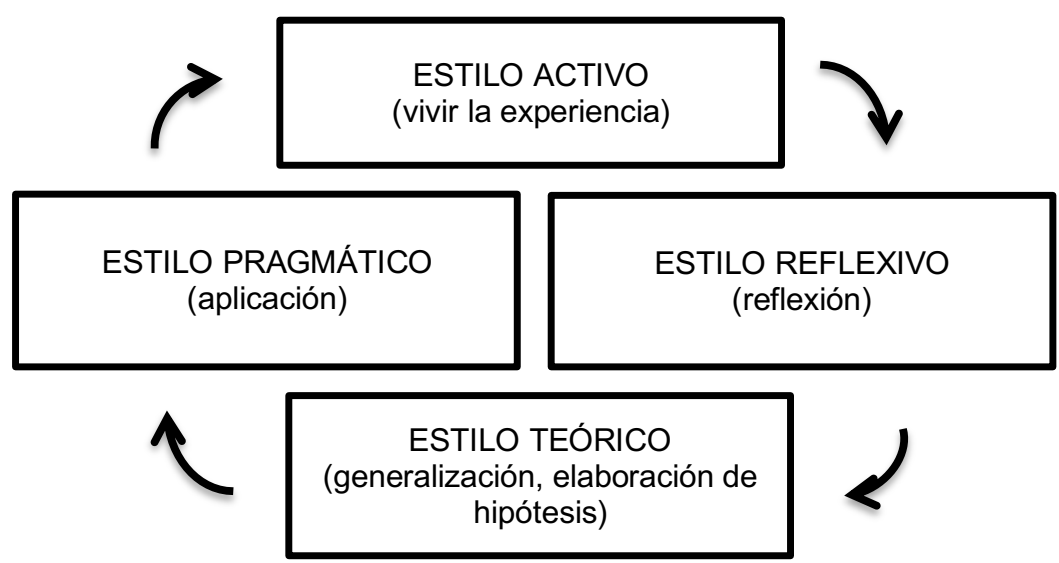

En consonancia, con estos estilos de aprendizaje y partiendo de que el estilo de enseñanza es un reflejo del propio estilo de aprendizaje, Martínez Geijo (2009) plantea una taxonomía de estilos de enseñanza dominante en el docente (Abierto, Formal, Estructurado y Funcional), que favorecerían los distintos estilos de aprendizaje. Es necesario, por tanto, producir estudios que muestren el emparejamiento del estilo de enseñanza del profesorado con el estilo de aprendizaje correspondiente del alumnado y su influencia en el rendimiento académico de estos últimos o, matching según Cuevas (2015).

\section{Método}

El objetivo general del estudio fue evaluar la incidencia de los estilos de aprendizaje, y su coincidencia con los estilos de enseñanza, sobre el rendimiento del alumnado frente a contenidos geográficos. Tratamos de dar respuesta a tres objetivos específicos con sus hipótesis de investigación asociadas. A saber:

Objetivo 1. Diagnosticar y describir el estilo de aprendizaje del alumnado participante, analizando sus perfiles según género y adscripción a un programa bilingüe españolinglés. (H1.1. . No existen diferencias según género, H1.1. Existen diferencias según género; H1.20. No existen diferencias en la adscripción a un programa bilingüe, H1.2. Existen diferencias en la adscripción a un programa bilingüe). 
Objetivo 2. Descubrir la relación existente entre los estilos de aprendizaje del alumnado y su rendimiento académico en Geografía. $\left(\mathrm{H}_{2}\right.$ No existe correlación, $\mathrm{H}_{2}$ Existe correlación).

Objetivo 3. Determinar la inferencia del ajuste entre los estilos de aprendizaje del alumnado y los estilos de enseñanza del profesorado en el rendimiento académico de los primeros en Geografía. (H3.10 No existen diferencias estadísticamente significativas, H3.1 Existen diferencias estadísticamente significativas).

El estudio asume una metodología cuantitativa no experimental o ex-post-facto, en cuanto que el investigador no tiene un control directo sobre las variables independientes 0 predictoras porque ya acontecieron sus manifestaciones o porque ya no pueden ser manipulables intrínsecamente. En particular, se adopta un diseño correlacional al hacer inferencias sobre relaciones, a partir de la variación común de las variables independientes y dependientes (Tabla 1), sin intervención directa. En este caso, tratamos de describir y analizar los efectos de determinadas variables predictoras de carácter personal y académico de alumnado y profesorado de Geografía e Historia en los perfiles estilísticos de aprendizaje de los primeros.

Tabla 1

Variables en el estudio

\begin{tabular}{|c|c|c|}
\hline & Variables predictoras/independientes & $\begin{array}{l}\text { Variables } \\
\text { criterio/dependientes }\end{array}$ \\
\hline \multirow{3}{*}{ Alumnado } & Género (Mujer/Hombre) & \multirow{3}{*}{ Estilos de aprendizaje } \\
\hline & Adscripción al programa bilingüe (Sí/No) & \\
\hline & Rendimiento académico del alumnado en Geografía & \\
\hline \multirow[t]{2}{*}{$\begin{array}{l}\text { Alumnado- } \\
\text { Profesorado }\end{array}$} & $\begin{array}{l}\text { Ajuste entre estilo de aprendizaje del alumnado y estilo } \\
\text { de enseñanza del profesorado en un emparejamiento }{ }^{1} \\
\text { determinado y en cualquier nivel de preferencia }{ }^{2} \\
\text { (Sí/No) }\end{array}$ & \multirow{2}{*}{$\begin{array}{l}\text { Rendimiento } \\
\text { académico del } \\
\text { alumnado en } \\
\text { Geografía }\end{array}$} \\
\hline & $\begin{array}{l}\text { Ajuste entre estilo de aprendizaje del alumnado y estilo } \\
\text { de enseñanza del profesorado en un emparejamiento }{ }^{1} \\
\text { y un nivel de preferencia }{ }^{2} \text { determinados (Sí/No) }\end{array}$ & \\
\hline
\end{tabular}

\footnotetext{
${ }^{1}$ Activo-Abierto, Reflexivo-Formal, Teórico-Estructurado y Pragmático-Funcional.

${ }^{2}$ Muy bajo, Bajo, Medio, Alto y Muy Alto.
}

Los instrumentos de recogida de información, además de los cuestionarios para destinados a la caracterización personal y académica del alumnado y el profesorado, abordan el diagnóstico de los estilos de enseñanza y de aprendizaje mediante el Cuestionario de Estilos de Enseñanza de Renes, Echeverry, Chiang, Rangel y Geijo (2013) y el Cuestionario de Estilos de Aprendizaje CHAEA-Junior (Sotillo Delgado, 2012, 2014); estos instrumentos se componen de 80 y 44 ítems, respectivamente, a los que el docente o el discente responde afirmativa 0 negativamente, agrupándose en cuatro dimensiones que se relacionan con cada uno de los estilos que se evalúan. 
La medida del rendimiento académico del alumnado en Geografía se lleva a cabo considerando las calificaciones finales obtenidas en la materia de Ciencias Sociales para tercer curso de Educación Secundaria Obligatoria. Cada centro participante aportó a la investigación las calificaciones finales del mes de junio, que se concretan cuantitativamente en números enteros que toman como valor mínimo el 1 y como valor máximo el 10.

La población de estudio es el alumnado de tercer curso de Educación Secundaria Obligatoria en centros educativos del municipio de Murcia (España). La selección de los participantes del estudio responde a un muestro no probabilístico casual o muestro por accesibilidad (Sabariego Puig, 2014). En la selección de los participantes se ha respetado un procedimiento de grupo intacto (por centros educativos) y tomando como factores de representatividad de la población objeto de estudio los siguientes: titularidad de los centros educativos de acuerdo con la población ( $60 \%$ de centros públicos y $40 \%$ de concertados-privados), laicidad educativa y tamaño muestral mínimo representativo de la población; este último sería de 356 estudiantes, aplicando los criterios de Morales Vallejo (2012).

Finalmente, la muestra del trabajo la componen diez centros educativos, seis públicos y cuatro privados-concertados, del municipio de Murcia (Región de Murcia-España). Participaron 21 docentes de Geografía e Historia y 483 estudiantes de tercer curso de Educación Secundaria Obligatoria (ESO).

\section{Resultados y discusión}

Se estructuran atendiendo a los objetivos del estudio y las hipótesis correspondientes a cada uno de ellos.

Diagnosticar y describir el estilo de aprendizaje del alumnado participante, analizando sus perfiles según género y adscripción a un programa bilingüe español-inglés

La respuesta al objetivo primero de investigación requiere determinar el perfil de estilos de aprendizaje del alumnado objeto de estudio, apreciándose diferencias de medias que son superiores en los estilos Reflexivo $(M=8.08, S D=1.92)$ y Teórico $(M=7.69, S D=1.91)$, respecto al Pragmático $(M=6.97, S D=1.77)$ y al Activo $(M=6.69, S D=1.89)$, como refleja la Figura 2.

Figura 2.

Perfil de aprendizaje del alumnado participante $(n=483)$

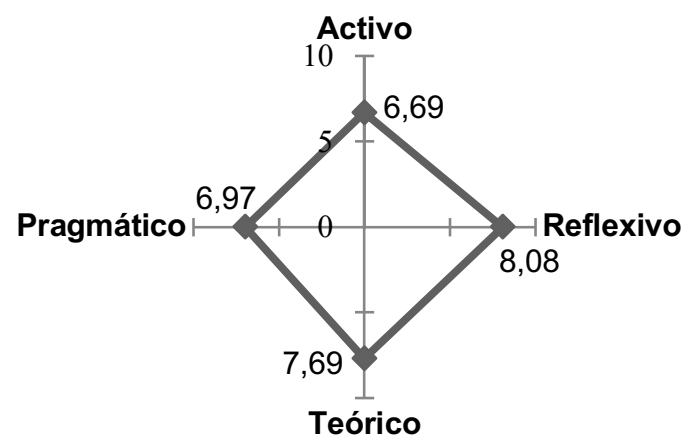

La interpretación del perfil estilístico de aprendizaje se realiza atendiendo a cinco niveles de preferencia, calculándose para nuestros participantes un baremo general abreviado de cada 
estilo, a través de los intervalos intersticiales de los percentiles 10, 30, 70 y 90: muy alta, alta, moderada, baja y muy baja (Alonso y otros, 1999). Los resultados arrojan los siguientes valores para cada estilo de aprendizaje: Activo $\left(P_{10}=4 ; P_{30}=6 ; P_{70}=8 ; P_{90}=9\right)$, Reflexivo $\left(P_{10}=5\right.$; $\left.P_{30}=7 ; P_{70}=9 ; P_{90}=10\right)$, Teórico $\left(P_{10}=5 ; P_{30}=7 ; P_{70}=9 ; P_{90}=10\right)$ y Pragmático $\left(P_{10}=5 ; P_{30}=6 ; P_{70}=8\right.$; $\left.P_{90}=9\right)$. En las Tablas 2 y 3 se muestran respectivamente, el baremo de interpretación y los descriptivos de frecuencia de los niveles de preferencia del alumnado de tercer curso de ESO para cada estilo de aprendizaje.

Tabla 2

Baremo general abreviado para interpretar los niveles preferencia de estilos de aprendizaje del alumnado $(\mathrm{n}=483)$

\begin{tabular}{lllll}
\hline \multirow{2}{*}{ Nivel de preferencia } & \multicolumn{4}{l}{ Puntuación en estilo de aprendizaje } \\
\cline { 2 - 5 } & Activo & Reflexivo & Teórico & Pragmático \\
\hline Muy alta (10\%) & $10-11$ & 11 & 11 & $10-11$ \\
Alta (20\%) & 9 & 10 & 10 & 9 \\
Moderada (40\%) & $7-8$ & $8-9$ & $8-9$ & $7-8$ \\
Baja (20\%) & $5-6$ & $6-7$ & $6-7$ & 6 \\
Muy Baja (10\%) & $0-4$ & $0-5$ & $0-5$ & $0-5$ \\
\hline
\end{tabular}

Tabla 3

Niveles de preferencia de los estilos de aprendizaje en el alumnado $(\mathrm{n}=483)$

\begin{tabular}{|c|c|c|c|c|c|c|c|c|c|c|c|c|}
\hline \multirow{2}{*}{$\begin{array}{l}\text { Nivel de } \\
\text { preferencia }\end{array}$} & \multicolumn{3}{|c|}{ Activo } & \multicolumn{3}{|c|}{ Reflexivo } & \multicolumn{3}{|c|}{ Teórico } & \multicolumn{3}{|c|}{ Pragmático } \\
\hline & $\mathbf{n}$ & $\%$ & $\sum \%$ & $\mathbf{n}$ & $\%$ & $\sum \%$ & $\mathbf{n}$ & $\%$ & $\Sigma \%$ & $\mathbf{n}$ & $\%$ & $\Sigma \%$ \\
\hline Muy alta (10\%) & 31 & 6.4 & 6.4 & 32 & 6.6 & 6.6 & 26 & $5 \cdot 4$ & $5 \cdot 4$ & 34 & 7.0 & 7.0 \\
\hline Alta (20\%) & 52 & 10.8 & 17.2 & 95 & 19.7 & 26.3 & 65 & 13.5 & 18.9 & 63 & 13.0 & 20.0 \\
\hline $\begin{array}{l}\text { Moderada } \\
(40 \%)\end{array}$ & 188 & 38.9 & 56.1 & 193 & 40.0 & 66.3 & 177 & 36.6 & 55.5 & 211 & 43.7 & 63.7 \\
\hline Baja (20\%) & 148 & 30.6 & 86.7 & 114 & 23.6 & 89.9 & 154 & 31.9 & 87.4 & 78 & 16.1 & 79.8 \\
\hline $\begin{array}{l}\text { Muy } \\
(10 \%)\end{array}$ & 64 & 13.3 & 100 & 49 & 10.1 & 100 & 61 & 12.6 & 100 & 97 & 20.1 & 100 \\
\hline
\end{tabular}

En nuestros estudiantes dominan los estilos de aprendizaje Reflexivo y Teórico, coincidiendo con el diagnóstico de Díaz-Serrano y Miralles (2016) con alumnado de tercer ciclo de Educación Primaria: el perfil parece conservar en ESO las dominancias estilísticas forjadas en la Primaria. Tales resultados coinciden con los de Ros, Cacheiro y Gallego (2017) que diagnostican como predominante el estilo Reflexivo en estudiantes de Bachillerato. Por su parte, Hervás (2008) estudia los estilos de aprendizaje del alumnado de tercer y cuarto curso de ESO, concluyendo que predomina el perfil de estudiantes que prefieren ambientes formales de trabajo, las actividades individualizadas y la interacción exclusiva con profesorado y adultos; preferencias de aprendizaje que nosotros asociamos a los estilos dominantes diagnosticados en nuestros estudiantes: Teórico y Estructurado. 
Recordemos aquí que, de acuerdo con Alonso y otros (1999) y Honey y Mumford (1988), el estilo Reflexivo se asocia a estudiantes que se centran en analizar y reflexionar sobre las experiencias, observan sin intervenir hasta dominar la situación y barajan todas las alternativas antes de tomar decisiones o sacar conclusiones. El estilo Teórico, cuya etapa de especialización es la de generalización-elaboración de hipótesis, se asocia a estudiantes que adaptan e integran sus observaciones dentro de teorías lógicas, enfocan los problemas por etapas, sintetizan para establecer principios, teorías y modelos y tienden al perfeccionismo y a buscar lo racional y objetivo, rechazando lo ambiguo.

A continuación, se aborda desde la estadística no paremátrica, el análisis inferencial para el contraste de las hipótesis contempladas para estudiar la influencia de variables sobre el perfil estilístico de aprendizaje del alumnado. Dado el incumplimiento del supuesto de normalidad en la distribución de los datos de las puntuaciones en cada uno de los cuatro estilos de aprendizaje, estamos en disposición de tomar la hipótesis alterna, asociada en este caso a una distribución distinta a la Normal según los resultados de la prueba Shapiro-Wilk $(n=483)$ y considerando cada uno de los factores que se contrastan en las hipótesis de investigación de este objetivo:

- Género: S-W $W_{\text {Activo-Mujer }}=.968, p<.001, S-W_{\text {Activo-Hombre }}=.971, p<.001 ; S-W_{\text {Reflexivo-Mujer }}=.929, p$ $<.001, \mathrm{~S}$-W $W_{\text {Reflexivo-Hombre }}=.936, p<.001 ; \mathrm{S}-W_{\text {Teórico-Mujer }}=.957, p<.001$, S-W $W_{\text {Teórico-Hombre }}=.961$, $p<.001 ; S-W_{\text {Pragmático-Mujer }}=.956, p<.001, S-W_{\text {Pragmático-Hombre }}=.961, p<.001$.

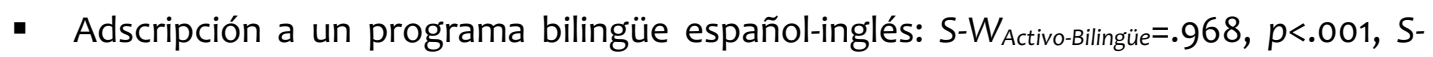
$W_{\text {Activo-Ordinario }}=.971, \quad p<.001 ; \quad S-W_{\text {Reflexivo-Bilingüe }}=.936, \quad p<.001, \quad S-W_{\text {Reflexivo-Ordinario }}=.926$, $p<.001 ; \quad S-W_{\text {Teórico-Bilingüe }}=.948, \quad p<.001, \quad S-W_{\text {Teórico-Ordinario }}=.957, \quad p<.001 ; \quad S-W_{\text {Pragmático- }}$ Bilingüe $=.949, p<.001, S-W$ Pragmático-Ordinario $=.962, p<.001$.

Centrándonos en la Hipótesis 1.1. Diferencias en los estilos de aprendizaje según género (con sus respectivas $\mathrm{H} 1.1_{0}-\mathrm{H} 1.1_{1}$ ), la Tabla 4 (más abajo) recoge los estadísticos descriptivos de las puntuaciones en los estilos de aprendizaje del alumnado participante, apreciándose diferencias de medias que son favorables a las mujeres en los estilos Activo, Reflexivo y Teórico, mientras que lo es para los hombres únicamente en el estilo de aprendizaje Pragmático.

La prueba de rangos $U$ de Mann-Whitney permite contrastar que no existe un efecto estadísticamente significativo de la variable de género del alumnado en los estilos de aprendizaje Activo $(U=27746.5, Z=-.740, p>.05)$ y Pragmático $(U=28390.5, Z=-.313, p>.05)$. Sin embargo, sí existen diferencias estadísticamente significativas en la distribución de las puntuaciones de los estilos Reflexivo $(U=25040.5, Z=-2.541, p<.05)$ y Teórico $(U=24632.5, Z=-$ $2.806, p=.05$ ) entre alumnos y alumnas. A juzgar por el análisis descriptivo previo, las mayores diferencias de medias favorables a las chicas se producen en los estilos cuya distribución presenta diferencias atendiendo al género; esto es, las alumnas participantes se distribuyen más homogéneamente que los alumnos en torno a puntuaciones más altas en los estilos Reflexivo y Teórico. Estamos pues en condiciones de aceptar la hipótesis alterna (H1.1 I $_{1}$ : existen diferencias en los estilos de aprendizaje entre alumnos y alumnas. Estos resultados son congruentes con Serrano Pastor (1994), quien apunta al género como buena variable predictora de los estilos de aprendizaje de estudiantes murcianos de ESO. Por el contrario, Hervás (2008) descarta la existencia de diferencias de género en ESO, como Sotillo Delgado (2012) y Díaz-Serrano y Miralles (2016) en Educación Primaria. 
Tabla 4

Descriptivos de puntuaciones estilísticas de aprendizaje según género $(n=483)$

\begin{tabular}{lllllllll}
\hline \multirow{2}{*}{ Género } & Estilos de & \multicolumn{2}{l}{ Estadísticos } & & & & \\
\cline { 5 - 8 } & aprendizaje & $\mathbf{n}$ & Rango & Mín & Máx & M & DS \\
\hline \multirow{2}{*}{ Mujer } & Activo & 217 & 10 & 1 & 11 & 6.75 & 1.904 \\
& Reflexivo & 217 & 9 & 2 & 11 & 8.35 & 1.776 \\
& Teórico & 217 & 8 & 3 & 11 & 7.99 & 1.777 \\
& Pragmático & 217 & 8 & 2 & 10 & 6.95 & 1.658 \\
\hline \multirow{2}{*}{ Hombre } & Activo & 266 & 10 & 1 & 11 & 6.64 & 1.873 \\
& Reflexivo & 266 & 10 & 1 & 11 & 7.87 & 2.015 \\
& Teórico & 266 & 11 & 0 & 11 & 7.44 & 1.988 \\
& Pragmático & 266 & 9 & 2 & 11 & 6.98 & 1.861 \\
\hline
\end{tabular}

En referencia a la Hipótesis 1.2. Diferencias en los estilos de aprendizaje según adscripción del alumnado al programa bilingüe español-inglés $\left(H 1.2_{0}-H 1.2_{1}\right)$, se muestran en la Tabla 5 los estadísticos descriptivos de las puntuaciones en los estilos de aprendizaje del alumnado participante, apreciándose diferencias de medias entre ambos grupos que toman valores muy bajos; esto es, el alumnado que cursa el programa educativo bilingüe y el que no lo hace presentan puntuaciones medias muy similares en todos los estilos de aprendizaje.

\section{Tabla 5}

Descriptivos de puntuaciones estilísticas de aprendizaje según adscripción del alumnado al programa bilingüe español-inglés $(n=483)$

\begin{tabular}{|c|c|c|c|c|c|c|c|}
\hline \multirow{2}{*}{$\begin{array}{l}\text { ¿Bilingüe } \\
\text { español- } \\
\text { inglés? }\end{array}$} & \multirow{2}{*}{$\begin{array}{l}\text { Estilos de } \\
\text { aprendizaje }\end{array}$} & \multicolumn{6}{|c|}{ Estadísticos } \\
\hline & & $\mathrm{n}$ & Rango & Mín & Máx & $\mathbf{M}$ & DS \\
\hline \multirow[t]{4}{*}{ Sí } & Activo & 176 & 9 & 2 & 11 & 6.70 & 1.735 \\
\hline & Reflexivo & 176 & 8 & 3 & 11 & 8.05 & 1.832 \\
\hline & Teórico & 176 & 11 & 0 & 11 & 7.63 & 1.826 \\
\hline & Pragmático & 176 & 8 & 2 & 10 & 6.92 & 1.719 \\
\hline \multirow[t]{4}{*}{ No } & Activo & 307 & 10 & 1 & 11 & 6.68 & 1.970 \\
\hline & Reflexivo & 307 & 10 & 1 & 11 & 8.11 & 1.977 \\
\hline & Teórico & 307 & 9 & 2 & 11 & 7.73 & 1.965 \\
\hline & Pragmático & 307 & 9 & 2 & 11 & 7.00 & 1.802 \\
\hline
\end{tabular}

La prueba de rangos $U$ de Mann-Whitney permite contrastar que no existe un efecto estadísticamente significativo del seguimiento de un programa bilingüe español-inglés sobre los estilos de aprendizaje: Activo ( $U=26979.5, Z=-.025, p>.05)$, Reflexivo ( $U=26072, Z=-.649$, $p>.05)$, Teórico $(U=26036.5, Z=-.672, p>.05)$ y Pragmático $(U=26493, Z=-.360, p>.05)$. Con ello, 
se acepta la hipótesis nula ( $\mathrm{H}_{1.30}$ ): no existen diferencias en los estilos de aprendizaje del alumnado que cursa estudios en programa bilingüe y el alumnado que no lo hace.

Descubrir la relación existente entre los estilos de aprendizaje del alumnado y su rendimiento académico en Geografía

El objetivo segundo de estudio nos lleva a contrastar la influencia que pueda tener el estilo de aprendizaje del alumnado de tercer curso de ESO sobre el rendimiento académico que obtiene en Geografía, planteándose como hipótesis $\mathrm{H}_{2}-\mathrm{H}_{2}$. Las calificaciones en esta etapa se calculan entre 1 y 10 puntos, y su análisis descriptivo arroja un valor medio $M=6.36$ $(D S=2.09)$.

Para determinar la correlación entre las variables implicadas, se calculó la prueba Rho de Spearman, tomando las puntuaciones absolutas obtenidas por el alumnado en cada estilo de aprendizaje y poniéndolas en relación con sus calificaciones en Geografía: $R_{\text {Activo }}=-.135, p<.01$; $R_{\text {Reflexivo }}=.128, p=.01 ; R_{\text {Teórico }}=.094, p=.05 ; R_{\text {Pragmático }}=.016, p>.05$. Los resultados de las pruebas anteriores muestran una correlación estadísticamente significativa con un nivel de confianza del $99 \%(\alpha=.01)$ entre las puntuaciones en Activo y Reflexivo y las calificaciones en Geografía, haciéndolo con signo negativo y positivo, respectivamente. El estilo Teórico, guarda una relación directa respecto al rendimiento académico y estadísticamente significativa a un nivel crítico $\alpha=.0$. La prueba para el estilo Pragmático no se revela como un predictor estadísticamente significativo para el rendimiento académico.

Según los resultados descritos, el perfil de aprendizaje del alumnado determina el rendimiento académico en Geografía en la medida en que este puntúe más en los estilos Reflexivo y Teórico y menos en el Activo: los estudiantes obtendrán mejores calificaciones al enfrentarse a contenidos geográficos cuanto menos Activos y más Reflexivos y Teóricos sean. Estamos así en condiciones de aceptar la hipótesis de investigación alternativa $\left(\mathrm{H}_{1}\right)$ que apoya la correlación existente entre calificaciones en Geografía-puntuaciones en los estilos de aprendizaje del alumnado.

Los estilos Teórico y Estructurado que nuestro estudio asocia a rendimientos más elevados resultan coherentes con Hervás y Miralles (2004), quienes respaldan que los estudiantes obtienen más puntuación en Ciencias Sociales cuando acceden al conocimiento de un modo racional, atraídos por el mundo de las ideas desde un entorno tranquilo y estructurado. Además, nuestros resultados apoyan las consideraciones realizadas por Kolb (1984) a partir de los estudios que le permitieron desarrollar el modelo del aprendizaje experiencial, señalando que la Geografía es una disciplina asimilativa que favorece la conceptualización abstracta y la observación reflexiva, siendo atractiva para personas con un estilo Asimilador (Healey, Kneale y Bradbeer, 2005). Se corresponde así, con una transición de estilos de aprendizaje Reflexivo-Teórico según el modelo de Alonso y otros (1999).

El papel de las puntuaciones altas en estilo de aprendizaje Activo, afectando negativamente sobre el rendimiento de los discentes frente a contenidos geográficos, también ha sido detectado en el estudio de Sotillo Delgado (2012) en Educación Primaria, existiendo una relación inversa entre las puntuaciones en estilo Activo y el rendimiento académico en la materia de Conocimiento del Medio Natural, Social y Cultural. El trabajo de Díaz-Serrano y Miralles (2016), también en Educación Primaria, documenta la tendencia a un mayor rendimiento en las unidades didácticas de Geografía, cuanto mayor dominio presente el estilo Teórico en el perfil de aprendizaje. Ello, contrasta, sin embargo, con nuestros resultados en Educación Secundaria con una tendencia inversa de correlación en el rendimiento en Geografía. 
En niveles universitarios, Healey y otros (2005) diagnostican el estilo de aprendizaje de estudiantes de Geografía en distintos países de habla inglesa, siendo mayoritariamente asimiladores, es decir, Reflexivo-Teóricos.

Determinar la inferencia del ajuste entre los estilos de aprendizaje del alumnado y los estilos de enseñanza del profesorado en el rendimiento académico de los primeros en Geografía

Este objetivo nos lleva a indagar en la influencia que puede tener la coincidencia del Estilo de Aprendizaje del estudiante y el Estilo de Enseñanza de su docente a un nivel de preferencia similar (descriptivos de frecuencia en la Tabla 6 ) sobre la calificación que obtiene el alumnado en Geografía. Para ello, previamente se ha diagnosticado el estilo de enseñanza del profesorado que imparte Geografía a los grupos discentes participantes. Los descriptivos de las puntuaciones de los Estilos Formal $(M=12.1, S D=2)$ y Funcional $(M=12, S D=2.57)$ permiten observar diferencias de medias que les son favorables respecto a los estilos Estructurado $(M=11.38, S D=2.31)$ y Abierto $(M=11, S D=2.72)$ del profesorado participante, ver Figura 3.

Figura 3.

Perfil de enseñanza del profesorado participante de Educación Secundaria.

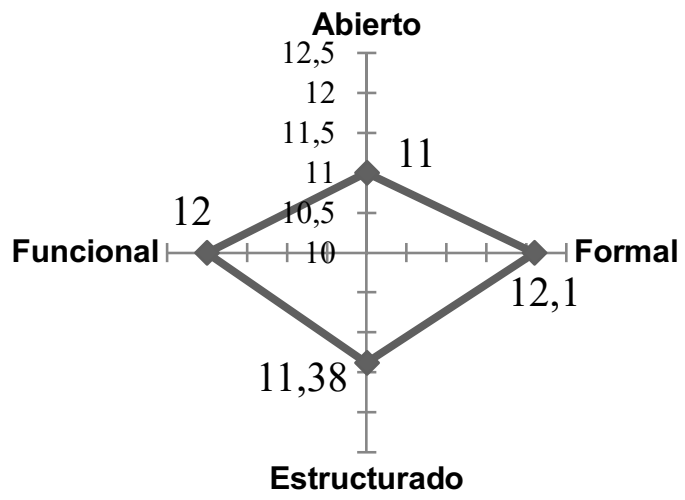

Las frecuencias de las coincidencias estilísticas halladas entre nuestros participantes para cada par de estilos en cualquiera de los niveles de preferencia son 107 estudiantes (22.2\%) para la relación entre Estilo de Aprendizaje Activo y Estilo de Enseñanza Abierto; 132 (27.3\%) entre Reflexivo y Formal; 144 (29.8\%) entre Teórico y Estructurado; y 105 (21.7\%) entre alumnado Pragmático y profesorado Funcional.

\section{Tabla 6}

Alumnado que presenta ajuste estilístico con su docente de Ciencias Sociales $(n=483)$, según niveles de preferencia

\begin{tabular}{l|ccc|ccc|ccc|ccc}
\hline \multirow{2}{*}{$\begin{array}{l}\text { Nivel de } \\
\text { preferencia }\end{array}$} & \multicolumn{3}{|c|}{ Activo-Abierto } & \multicolumn{3}{|c|}{$\begin{array}{c}\text { Reflexivo- } \\
\text { Formal }\end{array}$} & \multicolumn{2}{c|}{$\begin{array}{c}\text { Teórico- } \\
\text { Estructurado }\end{array}$} & \multicolumn{3}{c}{$\begin{array}{c}\text { Pragmático- } \\
\text { Funcional }\end{array}$} \\
\cline { 2 - 14 } & $\mathbf{n}$ & $\%$ & $\Sigma \%$ & $\mathbf{n}$ & $\%$ & $\sum \%$ & $\mathbf{n}$ & $\%$ & $\sum \%$ & $\mathbf{n}$ & $\%$ & $\sum \%$ \\
\hline Muy alta & 5 & 1 & 1 & 0 & 0 & 0 & 0 & 0 & 0 & 0 & 0 & 0 \\
Alta & 15 & 3.1 & 4.1 & 34 & 7 & 7 & 5 & 1 & 1 & 15 & 3.1 & 3.1 \\
Moderada & 56 & 11.6 & 15.7 & 66 & 13.7 & 20.7 & 103 & 21.3 & 22.3 & 70 & 14.5 & 17.6
\end{tabular}




\begin{tabular}{l|ccc|ccc|ccc|ccc} 
Baja & 15 & 3.1 & 18.8 & 32 & 6.6 & 27.3 & 28 & 5.8 & 28.1 & 14 & 2.9 & 20.5 \\
Muy Baja & 16 & 3.3 & 22.1 & 0 & 0 & 27.3 & 8 & 1.7 & 29.8 & 6 & 1.2 & 21.7 \\
No coincide & 376 & 77.8 & 100 & 351 & 72.7 & 100 & 339 & 70.2 & 100 & 378 & 78.3 & 100 \\
\hline TOTAL & 483 & 100 & & 483 & 100 & & 483 & 100 & & 483 & 100 & \\
\hline
\end{tabular}

Para tratar de dar respuesta a este objetivo, nos preguntamos si la coincidencia estilística o la falta de esta es una variable predictora del rendimiento académico en Geografía. La tabla 7 muestra los descriptivos de las calificaciones cuando coinciden y cuando no lo hacen en los distintos emparejamientos estilísticos.

Tabla 7

Calificaciones en Geografía del alumnado, según ajuste (o desajuste) estilístico con su docente de Geografía e Historia $(\mathrm{n}=483)$

\begin{tabular}{llllllllll}
\hline \multirow{2}{*}{ ¿Coincidencia? } & \multicolumn{2}{c}{ Activo-Abierto } & \multicolumn{2}{c}{ Reflexivo-Formal } & \multicolumn{2}{l}{$\begin{array}{l}\text { Teórico- } \\
\text { Estructurado }\end{array}$} & \multicolumn{2}{c}{$\begin{array}{l}\text { Pragmático- } \\
\text { Funcional }\end{array}$} \\
\cline { 2 - 10 } & $\mathbf{M}$ & SD & M & SD & M & SD & M & SD \\
\hline Sí & 6.24 & 1.93 & 5.94 & 2.18 & 6.35 & 2.21 & 6.25 & 2.04 \\
No & 6.39 & 2.14 & 6.52 & 2.04 & 6.36 & 2.04 & 6.39 & 2.11 \\
\hline
\end{tabular}

La prueba $U$ de Mann-Whitney arroja un valor estadísticamente significativo $(\alpha=.01)$ únicamente cuando coinciden estudiante Reflexivo-docente Formal en las clases de Geografía: $U=19631.5, Z=-2.618, p<.01:$ aceptamos la hipótesis alterna solo en esta relación estilística: H3.1 - Existen diferencias estadísticamente significativas entre las calificaciones que obtiene el alumnado en Geografía, según el ajuste estilístico con su docente. El resto de emparejamientos estilísticos no arrojan valores significativos: Activo-Abierto ( $U=19059, Z=-$ $.840, p>.05)$, Teórico-Estructurado ( $U=24102.5, Z=-.220, p>.05)$, Pragmático-Funcional $(U=18927, Z=-.735, p>.05)$.

Los datos indican que existen diferencias estadísticamente significativas $(\alpha=.01)$ en las calificaciones del alumnado en Geografía, según el nivel de preferencia Reflexivo-Formal, con la prueba $H$ de Kruskal-Wallis para $k$ muestras independientes $[H(3)=19.479, p<.01]$. Los estadísticos descriptivos de las calificaciones para cada uno de esos niveles de preferencia permiten detectar diferencias de medias que favorecen al nivel de preferencia Alta $(M=6.82$, $S D=1.98)$, nivel Moderado $(M=5.91, S D=2.36)$ y Bajo $(M=5.06, S D=1.60)$. Con ello, apoyamos los resultados de Serrano Pastor (1999): el emparejamiento del estilo de aprender y de enseñar correlaciona positivamente con el éxito académico. Nuestra experiencia empírica aporta evidencias de que las calificaciones del alumnado en Geografía mejoran cuando coinciden con profesorado de Geografía e Historia con preferencia alta por el estilo Reflexivo-Formal. Si como sabemos, la configuración del sistema educativo español favorece el rendimiento académico de los estudiantes reflexivos y teóricos, complementar los perfiles activos y pragmáticos con profesorado formal (Reflexivo) y estructurado (Teórico) podría permitir la flexibilización estilística mencionada, siendo esta la que conduzca al éxito académico por encima del emparejamiento estilístico. 


\section{Conclusiones}

Tras el análisis y la discusión de resultados esgrimidos, son tres las conclusiones a destacar:

1.-El perfil de aprendizaje del alumnado participante del municipio de Murcia está dominado por los estilos Reflexivo y Teórico en los que las alumnas alcanzan puntuaciones más elevadas que los alumnos. La adscripción a un programa bilingüe español-inglés no parece afectar a los estilos de aprendizaje. Si bien, diferentes investigaciones, entre ellas, el presente trabajo, destacan una continuidad en el alumnado al estudiar Geografía e Historia, en Educación Primaria y Secundaria, donde coinciden los perfiles con estos estilos de aprendizaje.

2.-Los estudiantes parece que obtienen calificaciones más elevadas frente a contenidos geográficos cuando su perfil de estilos de aprendizaje presenta puntuaciones más altas en Reflexivo y Teórico y más bajas en Activo.

3.-El emparejamiento congruente de estilos de enseñanza y de aprendizaje afecta positivamente a nuestro alumnado solo cuando se produce entre docentes formales y discentes reflexivos con niveles de preferencia altos: las calificaciones de un estudiante son mejores cuando coincide con su docente de Geografía e Historia en poseer una preferencia alta por los estilos Reflexivo y Formal, respectivamente.

Podemos decir, por tanto, coincidiendo con Alonso y colaboradores (1999) que, aunque los educadores se han preocupado tradicionalmente por dar respuesta a las necesidades de cada educando, otorgando importancia a la personalización de la enseñanza, sería preciso cambiar la perspectiva para superar el debate tradicional sobre la ratio docente/discente y los materiales didácticos, para poner el foco en un aspecto crucial: cómo aprende cada estudiante.

La información que nos aportan los estilos de aprendizaje permite conocer las características individuales y agrupar al alumnado, pudiendo ayudar al profesorado a plantear una variedad de actividades que apoyen al aprendizaje (Hawk y Shah, 2007; Jiménez, Vega, Capa, Fierro y Quichimbo, 2019). Aunque los estilos de aprendizaje pueden cambiar dependiendo de factores múltiples -edad, género, carrera, cultura o idioma- (Joy y Kolb, 2009; Maldonado, 2014; Villalba, 2015), conocerlos, aún no siendo la única solución para el éxito, permitirá al docente aplicar recursos educativos que contribuyan a diversificar la forma de aprender de cada estudiante, con las fortalezas y debilidades de su estilo preferente/dominante. de Jiménez y otros (2019) señalan al respecto mejoras en el rendimiento: "los alumnos con estilos de aprendizaje múltiples tienden a obtener mejor puntaje que aquellos en los que predomina un estilo de aprendizaje" (p. 8).

Más específicamente, se ha documentado que el profesorado de Geografía e Historia en Educación Secundaria suele enseñar mediante actividades tradicionales estructuradas formalmente, con la lección magistral y las tareas escritas y orales centradas en lecturas como protagonistas (Molina, Miralles, Deusdad y Alfageme, 2017). Si bien, las habilidades narrativas de estudiantes que se están formando como maestros suelen ser pobres y su nivel de alfabetización histórica muy bajo (Gómez y Sain, 2017). Se olvida el aprendizaje a partir de experiencias, en pro de un aprendizaje planteado en términos de adquisición de conceptos que nos resulta más familiar (Honey y Mumford, 1988). Sin embargo, para De Miguel (2015) el aprendizaje experiencial se puede potenciar con la enseñanza de la Geografía, a través de actividades más creativas e innovadoras: por ejemplo, con las Tecnologías de la Información que permiten adquirir un conocimiento geográfico estructurado en las ramas de la disciplina referente (Geografía Física, Humana, Regional). 
Asimismo, se ha documentado que el alumnado, cuando cambia de etapa educativa, inicialmente recurre a un estilo de aprendizaje previamente estructurado durante su historia académica, pero tras un determinado tiempo de estudio, algunos tienden a incorporar 0 ajustar ciertas estrategias y características de aprendizaje que derivan en un nuevo estilo, adecuándose a las necesidades de aprendizaje (González y Diago, 2019). En la misma línea, González-Peiteado y Pino-Juste (2016) defienden el principio de integración de estilos y flexibilidad, que refuerza el uso de estilos de enseñanza mixtos, aunque uno de ellos sea el preferente, para ofrecer oportunidades a todo estudiante y respetar las diferencias individuales. Defienden que:

\begin{abstract}
Se hace imprescindible que el futuro docente sea formado en metodologías activas y conducido en la búsqueda de estilos de enseñanza que le dote de los mecanismos necesarios para ser un verdadero agente de cambio, y a su vez, lo faculte para interactuar dentro de un marco de colaboración y actuación que le posibilite la toma de decisiones concretas que conecten con un espacio multicultural y cambiante (p. 1188)
\end{abstract}

En suma, y de acuerdo con Luengo y González (2005) en el contexto español, "nuestro sistema educativo no es neutro" (p. 160), favorece a los alumnos teóricos y reflexivos. Además, el alumnado pragmático puede aprovechar sus capacidades en algunas asignaturas, pero el ritmo que se impone a las actividades no les suele dar el tiempo que necesitan para elaborar y matizar ideas. Los resultados de nuestro estudio indican una prevalencia del alumnado de estilos teórico y reflexivo frente a la concreción didáctica que se hace de la materia en Geografía, en consonancia con los perfiles que favorece el sistema educativo. Todo apunta pues a la necesidad de un cambio en las metodologías de enseñanza y en los sistemas de evaluación para que los estudiantes con distintos estilos de aprendizaje no se queden fuera del sistema o, como señalaba Gallego (2013) para que el sistema educativo no haga propensos al fracaso escolar a estudiantes activos y pragmáticos

\title{
Referencias
}

Alonso, C. M., Gallego, D. J. y Honey, P. (1999). Los Estilos de Aprendizaje. Procedimientos de diagnóstico y mejora. Bilbao: Ediciones Mensajero.

Camana, R. G. y Torres, R. A. (2018). Descubrimiento del estilo de aprendizaje dominante de estudiantes de la carrera de Tecnología en Análisis de Sistemas. Revista Educación, 42(2), 1-13. https://doi.org/10.15517/revedu.v42i2.26473

Coto, M. (2020). Descubrimiento del estilo de aprendizaje dominante en estudiantes de $\begin{array}{llll}\text { Matemática Superior. Revista 1-13. } & \text { Educación, }\end{array}$ https://doi.org/10.15517/revedu.v44i1.38571

Cuevas, J. (2015). Is learning styles-based instruction effective? A comprehensive analysis of recent research on learning styles. Theory and Research in Education, 13(3), 308-333.

De Miguel, R. (2015). Del pensamiento espacial al conocimiento geográfico a través del aprendizaje activo con tecnologías de la información geográfica. Giramundo, 2(4), 713.

Delgado, U., Martínez, F. G. y Ponce, D. J. (2016). Análisis crítico de los estilos de aprendizaje desde una perspectiva naturalista. Revista iberoamericana de psicología: ciencia y tecnología, 9(1), 45-52 
Díaz-Serrano, J. (2018). Caracterización de los estilos de enseñanza y de aprendizaje en geografía: un avance hacia su inclusión en las aulas de secundaria. Tesis doctoral. Universidad de Murcia, España.

Díaz-Serrano, J. y Miralles, P. (2016). La geografía en el Tercer Ciclo de Educación Primaria: estilos de aprendizaje y rendimiento académico. Boletín de la Asociación de Geógrafos Españoles, 72, 447-469.

Gallego, D. J. (2013). Ya he diagnosticado el estilo de aprendizaje de mis alumnos y ahora ¿qué hago? Revista de Estilos de Aprendizaje, 6(12), 1-15.

Gómez, C. J. y Saiz, J. (2017). Investigación narrativa y habilidades históricas. Un estudio sobre la formación de maestros. REDIE, 19(4), 19- 33.

González, P. y Diago, M. L. (2019). Los estilos de aprendizaje: su utilidad en las aulas y herramientas de detección adecuadas. En R. Roig-Vila (Ed.), Investigación e innovación en la Enseñanza Superior. Nuevos contextos, nuevas ideas (pp. 194-203). Barcelona: Octaedro.

González-Peiteado, M. y Pino-Juste, M. (2016). Los estilos de enseñanza: construyendo puentes para transitar las diferencias individuales del alumnado. Revista Complutense de Educación, 27(3), 1175-1191.

Hawk, T. F. y Shah, A. J. (2007). Using learning style instruments to enhance student learning. Decision Sciences Journal of Innovative Education, 5(1), 1-19.

Healey, M., Kneale, P. y Bradbeer, J. (2005). Learning styles among geography undergraduates: an international comparison. Area, 37(1), 30-42.

Hervás, R. M. (2008). Identificación de variables que influyen en los estilos de aprendizaje. Claves para conocer cómo aprenden los estudiantes. Revista de Estilos de Aprendizaje, 1(1), 143-167.

Hervás, R. M., y Miralles, P. (2004). Preferencias y logro en el aprendizaje de las Ciencias Sociales. En C.M. Alonso y D.J. Gallego (Eds.), $1^{\text {er }}$ Congreso Internacional de Estilos de Aprendizaje. Actas (pp. 1-12). Madrid: Anaya/Universidad Nacional de Educación a Distancia.

Honey, P. y Mumford, A. (1988). The Manual of Learning Styles. Berkshire: Honey.

Isaza, L. y Henao, G. C. (2012). Attitudes - styles of education: relation with the academic performance. International Journal of Psychological Research, 5(1), 133-141. https://doi.org/10.21500/20112084.769

Jiménez, L. S., Vega, N., Capa, E. D., Fierro, N. C. y Quichimbo, P. (2019). Estilos y estrategia de enseñanza-aprendizaje de estudiantes universitarios de la Ciencia del Suelo. REDIE, 21(e04), 1-10.

Joy, S. y Kolb, D. A. (2009). Are there cultural differences in learning style? International Journal of Intercultural Relations, 33(1), 69-85.

Kolb, D. A. (1984). Experiential learning: experience as the source of learning and development. Englewood Cliffs, NJ: Prentice Hall.

Lambert, D. y Balderstone, D. (2010). Learning to teach Geography in the Secondary School. New York, NW: Routledge. 
Luengo, R. y González, J. J. (2005). Relación entre los estilos de aprendizaje, el rendimiento en matemáticas y la elección de asignaturas optativas en alumnos de E.S.O. RELIEVE, $11(2), 147-165$.

Laudadío, J. y Mazzitelli, C. (2019). Formación del profesorado: estilos de enseñanza y habilidades emocionales. Revista Mexicana de Investigación Educativa (RMIE), 24(82), 853-869.

Maldonado, S. (2014). Differences between Latino Students' Learning Styles and their gender. Institute for Learning Styles Journal, 1, 28-41.

Martínez Geijo, P. (2009). Estilos de enseñanza: conceptualización e investigación (en función de los estilos de aprendizaje de Alonso, Gallego y Honey). Revista de Estilos de Aprendizaje, 2(3), 3-19.

Molina, S., Miralles, P., Deusdad, B. y Alfageme, M. B. (2017). Enseñanza de la historia, creación de identidades y prácticas docentes. Profesorado, 21(2), 331-354.

Morales Vallejo, P. (2012). Estadística aplicada a las Ciencias Sociales. Tamaño necesario de la muestra. ¿Cuántos sujetos necesitamos? Manuscrito no publicado. http://www.upcomillas.es/personal/peter/investigacion/Tama\%F1oMuestra.pdf

Nivela-Cornejo, M. A., Echeverría-Desiderio, S. V. y Morillo, R. (2019). Estilos de aprendizaje y rendimiento académico en el contexto universitario. Dominio de las Ciencias, 5(1), 70104.

Pérez, M. G., Leyva, H. P., Ocampo, F. y De Luna, R. (2018). Propuesta de estrategias de aprendizaje acorde a los estilos de aprendizaje de los estudiantes de Ingeniería de la ESCOM del IPN. RIDE (Revista Iberoamericana para la Investigación y el Desarrollo Educativo), 9(17), 1-26.

Renes, P., Echeverry, L.M., Chiang, M.T., Rangel, L. y Geijo, P.M. (2013). Estilos de Enseñanza: un paso adelante en su conceptualización y diagnóstico. (En función de los Estilos de Aprendizaje de Alonso, Gallego y Honey, 1994). Revista de Estilos de Aprendizaje, 6(11), 4-18.

Renés, P. y Martínez, P. (2015). Estilos de enseñanza y aprendizaje. Conceptualizaciones, investigaciones y orientaciones para la práctica educativa. Bilbao: Ediciones Mensajero.

Ros, N., Cacheiro, M. L. y Gallego, D. J. (2017). Preferencias en estilos de aprendizaje de los alumnos que cursan los estudios de bachillerato en la región de Murcia. Tendencias Pedagógicas, 30, 105-116.

Ruiz, B. L., Trillos, J. y Morales, J. (2006). Estilos de aprendizaje y rendimiento académico en estudiantes universitarios. Revista Galego-Portuguesa de Psicoloxía e Educación, 1112(13), 441-457.

Sabariego Puig, M. (2014). El proceso de investigación (parte 2). En R. Bisquerra (coord..), Metodología de la investigación educativa (pp. 127-163). Madrid: La Muralla.

Serrano Pastor, F. J. (1994). Evaluación de la interacción de los estilos de enseñanza y de aprendizaje en contextos escolares. Tesis Doctoral. Universidad de Murcia (España).

Sotillo Delgado, J. F. (2012). Los estilos de aprendizaje en alumnos de Primaria. Diagnóstico y propuesta pedagógica. Tesis doctoral. Universidad Nacional de Educación a Distancia, UNED, España. 
Sotillo Delgado, J.F. (2014). El cuestionario CHAEA-Junior o cómo diagnosticar el estilo de aprendizaje en alumnos de Primaria y Secundaria. Journal of Learning Styles, 7(13), 182201.

Stobart, G. (2010). Tiempos de pruebas: los usos y abusos de la evaluación. Madrid: Ediciones Morata/Ministerio de Educación, Gobierno de España.

Villalba, A. B. (2015). Estilos de aprendizaje en alumnos universitarios de profesorado en biología y licenciatura en biodiversidad. Revista de Estilos de Aprendizaje, 8(16), 79100. 